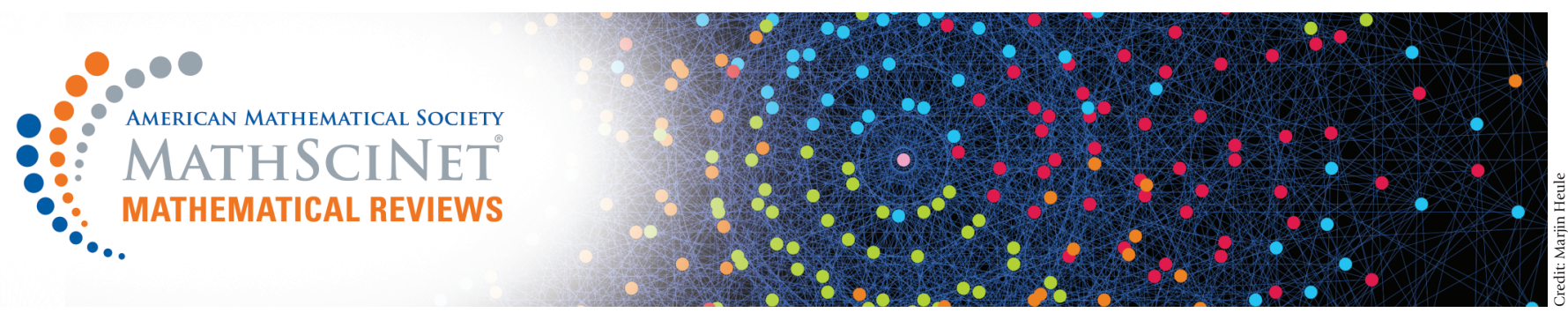

\title{
Who are you?
}

\section{Edward Dunne}

Mathematical Reviews is not just a bibliographic database of the mathematical literature; it also contains reviews of many of the items in the database. The reviews have been a hallmark of the operation from the very beginning. While the full-time staff of Mathematical Reviews puts in a lot of work to produce what is now MathSciNet ${ }^{\circledast}$, the expertise of the many reviewers is an essential part of what makes Mathematical Reviews exceptional. As of this writing, we have 22,652 active reviewers.

The list of the 285 reviewers in the first volume (covering all of 1940, the first year of publication) is quite impressive. It reads like a who's who of mathematics from the time. The reviewers include at least one Nobel Laureate, one Fields Medalist (the first two medals were awarded in 1936, then not again until 1950), and several future Wolf Prize winners. There are 15 AMS presidents in the list, plus the father of an AMS President. You can find it here: https: // www. ams.org/publications/math-reviews /reviewersvolume1.

The list of all reviewers continues to include remarkable mathematicians. Here are some award-winning mathematicians who are reviewers:

- Fields Medals: 30 of 62 medalists have written at least one review. The most prolific are Michael Atiyah (229 reviews), Pierre-Louis Lions (218 reviews), and Lars Ahlfors (170 reviews). Of the most recent medalists (2018), Alessio Figalli has written the most reviews (28). Of

Edward Dunne is the Executive Editor of Mathematical Reviews at the American Mathematical Society. His email address is egd@ams .org.

For permission to reprint this article, please contact: reprint -permission@ams.org.

DOI: https://dx.doi.org/10.1090/noti 1877 the medalists in this century, Cédric Villani is the most prolific reviewer, having written 98 reviews.

- Abel Prize: 16 of the 19 laureates have written reviews. The most prolific are Michael Atiyah, Srinivasa Varadhan (106 reviews), and Mikhail Gromov (101 reviews).

- Steele Prize for Lifetime Achievement: 22 of the 29 prizewinners have written at least one review. The most prolific are Henry P. McKean (315 reviews), Harry Kesten (262 reviews), Frederick Gehring (159 reviews), and Ralph S. Phillips (128 reviews).

- Steele Prize for Seminal Contribution: 19 of the 38 prizewinners have written at least one review. The most prolific are Louis de Branges (257 reviews), Srinivasa Varadhan, and Mikhael Gromov.

\section{Counting Reviews}

A frequent question we receive from new reviewers or prospective reviewers is "How many reviews do people normally write in a year?" Well, as a database, we have the data. From 2000 to 2017 (the last year for which we have complete data at this time), the median number of reviews written per year (per reviewer) has held steady at 3. The average number of reviews has varied from 3.17 to 4.45 , with the low coming in 2016 and the high in 2005. From this you might deduce (correctly) that we have some reviewers who write a lot of reviews. There are 20 reviewers who are still active and who have written 500 or more reviews: 


\section{Current Prolific Reviewers:}

Bucki, Andrew 1244

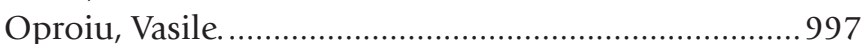

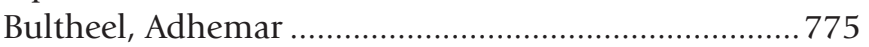

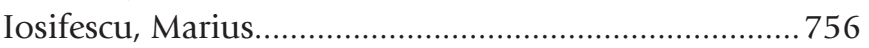

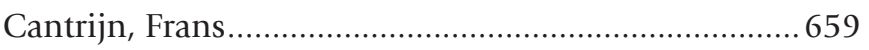

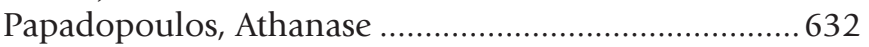

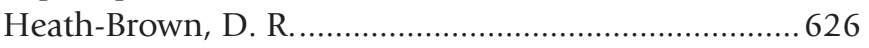

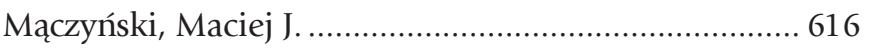

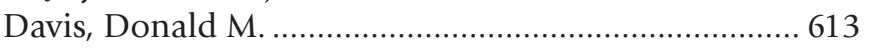

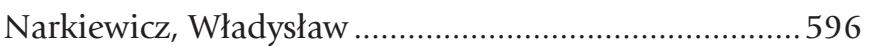

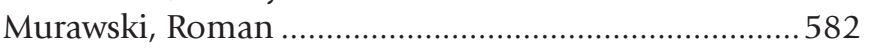

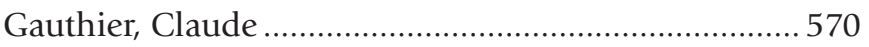

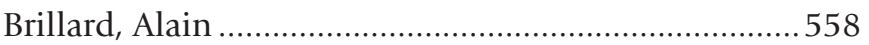

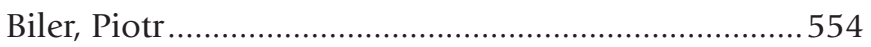

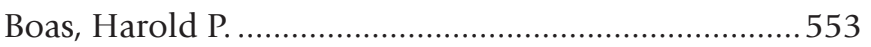

Hušková, Marie ………………………………............ 553

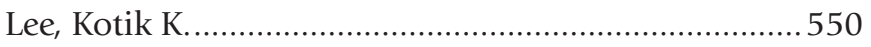

Borges, Manoel F. ....................................................... 527

Böttcher, Albrecht..................................................... 522

Moon, John W. .......................................................... 521

Historically, we also had some rather prolific reviewers, some of whom you may recognize:

\section{Some Prolific Past Reviewers:}

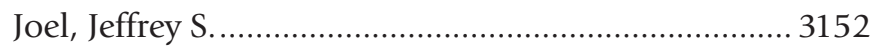

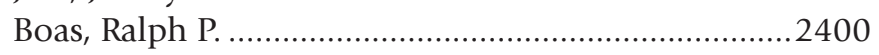

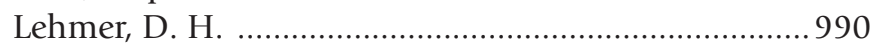

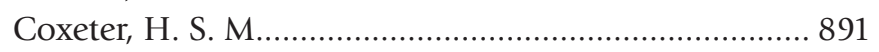

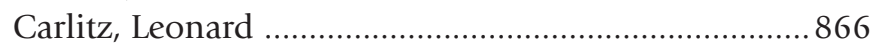

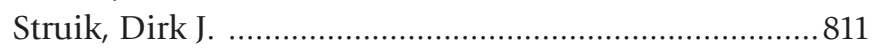

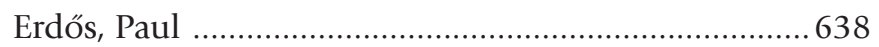

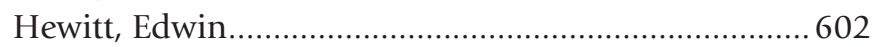

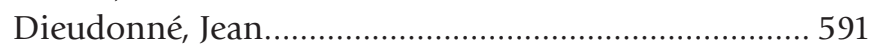

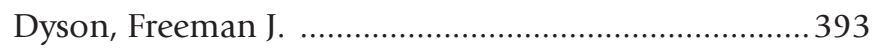

Niven, Ivan ............................................................... 361

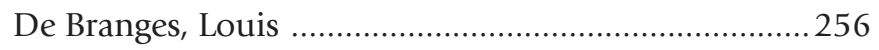

Fleming, Wendell H. ................................................... 192

The name Jeffrey Joel may not be familiar to many. He was an Associate Editor at Mathematical Reviews from 1973 to 1991. Ralph Boas, who has several claims to fame, was the Executive Editor of Mathematical Reviews from 1945 to 1950. His son, Harold, is keeping up the family tradition of writing reviews, appearing on the list of current prolific reviewers.

The reviewers come from all over the world. We have active reviewers from 141 different countries. (For comparison, the United Nations has 193 member states.) About one-sixth come from the United States. Half of the reviewers come from six countries: United States, China, Italy, India, France, and Germany. Three quarters of the reviewers come from 16 countries: the above six plus Spain, Japan, Brazil, England, Iran, Turkey, Poland, Russia, Canada, and Romania.

\section{Reviewers and AMS Members}

Mathematical Reviews is a service of the American Mathematical Society. So, what is the correlation between membership and reviewing? Overall, about $10 \%$ of AMS members are active reviewers. This number is misleading, however, since the count of "all AMS members" used to compute this includes all the undergraduate and graduate student members, who are unlikely to be reviewers. About $17 \%$ of regular AMS members are reviewers. By category, the affiliate members (those living in a country classified by the World Bank as a low-income, lower middle-income, or upper middle-income economy) have the greatest percentage who are reviewers: $53 \%$. The next is reciprocity members (those who also belong to one of the foreign societies with which the AMS has established a reciprocity agreement), of whom a bit over $27 \%$ are reviewers.

\section{Reviewers by Subject Area}

Reviewers are not evenly distributed among the subject areas. In particular, we tend to have more reviewers in traditional areas of pure mathematics than in applied areas. Most reviewers are willing to review in multiple classes, so it is hard to pigeonhole someone as a "calculus of variations" reviewer, since they might also write reviews of papers in functional analysis or PDEs. So, there is a thinning out factor that might be necessary. Also, some reviewers are willing (and able) to write more reviews per year than others. Finally, it is not always clear what is pure mathematics or applied mathematics just by a 2-digit class. For instance, I'm about to count MSC 35 (PDEs) as "pure" and MSC 60 (probability) as "applied," but neither characterization seems fair. Nevertheless, counting the number of reviewers who have listed a particular 2-digit class gives an idea of how hard or easy it is to match a reviewer to a paper in that class. By this rough estimate, we have about four times the density of reviewers in the classes from 03 to 58 as in the classes from 60 to 86. Mathematical Reviews is always looking for reviewers in all areas, but you can see why we would be particularly happy to have more reviewers in these "applied areas," which leads us to the next section.

\section{Becoming a Reviewer}

How do you become a reviewer? Statistically, most reviewers are suggested by our editors. We contact them to ask if they are interested. Normally, we look for someone who has already published some papers, which gives us an idea of the person's research interests. The rule of thumb (a rough guideline) is to have three papers in MathSciNet. If you haven't been contacted by us, or if you were contacted, said "No," and have now changed your mind, then you can volunteer to become a reviewer by writing to math- 
rev@ams . org. If you are going to volunteer, it is best to include your MR Author ID. Find it by searching from the URL: https://mathscinet . ams .org/mathscinet /MRAuthorID/search. This link works whether your institution has a subscription to MathSciNet or not. In the block at the top of the page, you will see your MR Author ID. Mine is 239650. Note: if you don't have any publications in the Mathematical Reviews database, then you won't have an MR Author ID.

Why should you become a reviewer? One reason is a sense of duty: helping out your fellow mathematicians. Another reason is to keep up on mathematics. As a reviewer, you would be sent papers on topics related to your own interests. Often, these might be papers you already know about. However, they may also be papers you haven't heard about. As a reviewer myself, I found this second reason the more compelling. Reviewing was a great way to keep up on the literature. Now that I am Executive Editor, I don't write reviews any more, but I do miss it.

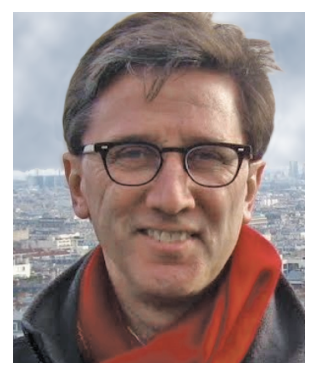

Edward Dunne

\section{Credits}

Author photo is courtesy of Edward Dunne.
LEARN ABOUT
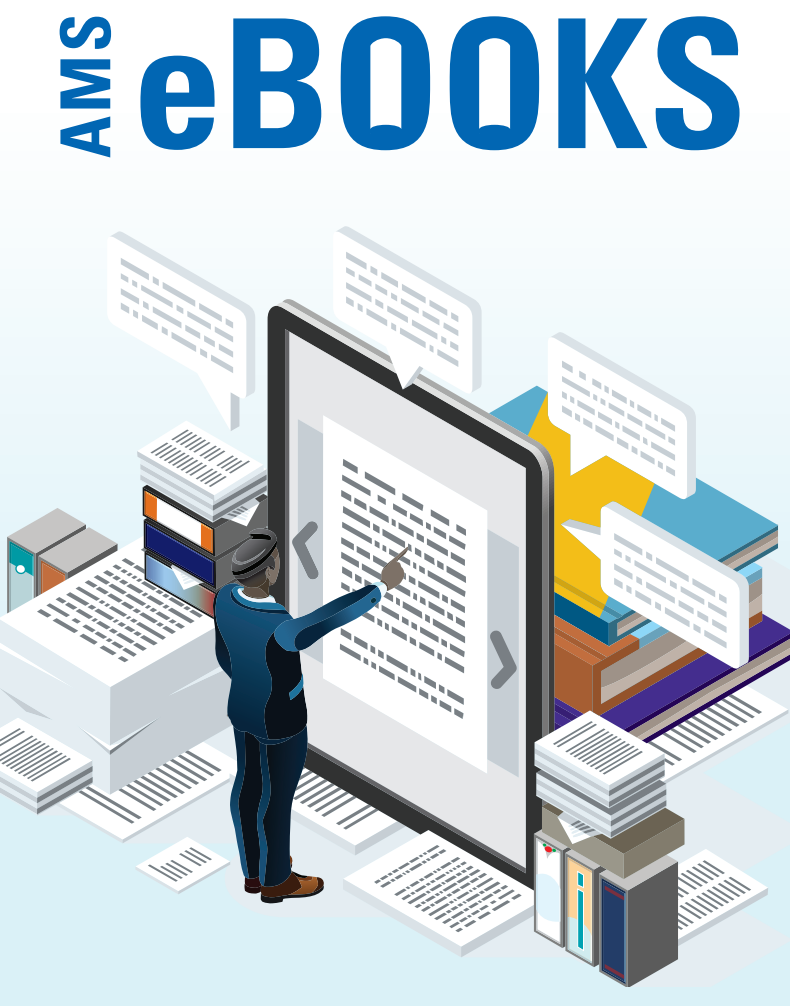

Did you know that most

of our titles are now

available in eBook form?

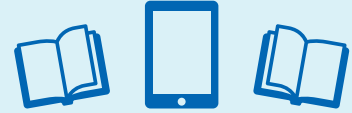

Browse both our print and electronic titles at bookstore.ams.org. 